\title{
INTUITIONISTIC LOGIC WITH A GALOIS CONNECTION HAS THE FINITE MODEL PROPERTY*
}

\author{
WOJCIECH DZIK, JOUNI JÄRVINEN, AND MICHIRO KONDO
}

\begin{abstract}
We show that the intuitionistic propositional logic with a Galois connection (IntGC), introduced by the authors, has the finite model property.
\end{abstract}

\section{INTRODUCTION}

In [1], we introduced the intuitionistic propositional logic with a Galois connection (IntGC). In addition to the intuitionistic logic axioms and inference rule of modus ponens, IntGC contains just two rules of inference mimicking the condition defining Galois connections. A Galois connection between partially ordered sets $P$ and $Q$ consists of two maps $f: P \rightarrow Q$ and $g: Q \rightarrow P$ such that for all $a \in P$ and $b \in Q$, we have $f(a) \leq b$ if and only if $a \leq g(b)$. Note that in the literature can be found two ways to define Galois connections - the one adopted here, in which the maps are order-preserving, and the other, in which they are reversing the order.

We proved in [1] that IntGC is complete with respect to both Kripke style and algebraic semantics. Our intention was also to show that IntGC has the finite model property (FMP), that is, for every formula which is not provable, there exists a finite counter Kripke model. Together with the other results presented in the paper, this would imply that the following assertions are equivalent for every IntGC-formula $A$ :

(i) $A$ is provable.

(ii) $A$ is valid in any finite distributive lattice with an additive and normal operator $f$;

(iii) $A$ is valid in any finite distributive lattice with a multiplicative and co-normal operator $g$;

(iv) $A$ is valid in any finite Kripke model for IntGC.

Unfortunately, our proof of FMP presented in [1] is incomplete and has some faults. For instance, we did not show that the frame on which the filtration is defined really forms a required Kripke frame. Therefore, here we present a more complete proof based on improved filtration model.

The paper is organised as follows. In Section 2, we recall the syntax, Kripke semantics and Kripke completeness of IntGC. Section 3 is devoted to proving the finite model property of IntGC.

${ }^{\star}$ Addendum to the article: Wojciech Dzik, Jouni Järvinen, and Michiro Kondo, Intuitionistic propositional logic with Galois connections, Logic Journal of the IGPL 18 (2010), 837-858. 


\section{LoGiC IntGC}

The language $\mathcal{L}$ of IntGC is constructed from a countable set of propositional variables $P$ and the connectives $\neg, \rightarrow, \vee, \wedge, \boldsymbol{\Lambda}, \nabla$. The constant true is defined by $\top:=p \rightarrow p$ for some fixed propositional variable $p \in P$, and the constant false is defined by $\perp:=\neg \top$.

The logic IntGC is the smallest logic in $\mathcal{L}$ that contains the intuitionistic propositional logic Int, and is closed under the rules of substitution, modus ponens, and the rules:

(GC1) If $A \rightarrow \nabla B$ is provable, then $\boldsymbol{\Delta} A \rightarrow B$ is provable.

(GC2) If $\boldsymbol{\Delta} A \rightarrow B$ if provable, then $A \rightarrow \nabla B$ is provable.

It is known that the following rules are admissible in IntGC:

(r1) If $A$ is provable, then $\nabla A$ is provable.

(r2) If $A \rightarrow B$ is provable, then $\nabla A \rightarrow \nabla B$ and $\boldsymbol{\Delta} A \rightarrow \boldsymbol{\Delta} B$ are provable.

In addition, the following formulas are provable:

(f1) $A \rightarrow \nabla \mathbf{\Delta} A$ and $\boldsymbol{\Delta} \nabla A \rightarrow A$.

(f2) $\boldsymbol{\Delta} A \leftrightarrow \boldsymbol{\Delta} \nabla \boldsymbol{\Delta} A$ and $\nabla A \leftrightarrow \nabla \mathbf{\Delta} \nabla A$.

(f3) $\nabla \top$ and $\neg \mathbf{\Lambda} \perp$.

(f4) $\nabla(A \wedge B) \leftrightarrow \nabla A \wedge \nabla B$ and $\boldsymbol{\Delta}(A \vee B) \leftrightarrow \boldsymbol{\Delta} A \vee \boldsymbol{\Delta} B$.

(f5) $\nabla(A \rightarrow B) \rightarrow(\nabla A \rightarrow \nabla B)$.

A structure $\mathcal{F}=(X, \leq, R)$ is called a Kripke frame of IntGC, if $X$ is a non-empty set, $\leq$ is a preorder on $X$, and $R$ is a relation on $X$ such that

$$
(\geq \circ R \circ \geq) \subseteq R .
$$

Let $v$ be a function $v: P \rightarrow \wp(X)$ assigning to each propositional variable $p$ a subset $v(p)$ of $X$. Such functions are called valuations and the pair $\mathcal{M}=(\mathcal{F}, v)$ is called an IntGC-model. For any $x \in X$ and $A \in \Phi$, we define a satisfiability relation in $\mathcal{M}$ inductively by the following way:

$$
\begin{aligned}
x \models p & \Longleftrightarrow x \in v(p), \\
x \models A \wedge B & \Longleftrightarrow x \models A \text { and } x \models A, \\
x \models A \vee B & \Longleftrightarrow x \models A \text { or } x \models A, \\
x \models A \rightarrow B & \Longleftrightarrow \text { for all } y \geq x, y \models A \text { implies } y \models B, \\
x \models \neg A & \Longleftrightarrow \text { for no } y \geq x \text { does } y \models A, \\
x \models \Delta A & \Longleftrightarrow \text { exists } y \text { such that } x R y \text { and } y \models A, \text { and } \\
x \models \nabla A & \Longleftrightarrow \text { for all } y, y R x \text { implies } y \models A .
\end{aligned}
$$

Let $x \leq y$. If $x \models \boldsymbol{\Delta} A$, there exists $z$ such that $x R z$ and $z \models A$. Now $y \geq x, x R z$, and $z \geq z$ imply $y R z$ by $(\star)$. Thus, $y \models \Delta A$. Similarly, if $y \not \models \nabla A$, then there exists $z$ such that $z R y$ and $z \not \models A$. Now $z \geq z, z R y$, and $y \geq x$ imply $z R x$. This means $x \not \forall \nabla A$. Hence, the frame is persistent.

An IntGC-formula $A$ is valid in a Kripke model $\mathcal{M}$, if $x=A$ for all $x \in X$. The formula is valid in a Kripke frame $\mathcal{F}$, if $A$ is valid in every model based on $\mathcal{F}$. The formula $A$ is Kripke valid if $A$ is valid in every frame.

We proved in [1] that every formula is Kripke valid if and only if it is provable. 


\section{IntGC HAS FMP}

Let $A$ be a formula that is not provable. Then, there exists a Kripke model $\mathcal{M}=(X, \leq, R)$ such that $A$ is not valid in $\mathcal{M}$. We construct a counter model for $A$ on a finite frame.

Let $\operatorname{Sub}(A)$ be the set of subformulas of $A$. We define the set

$$
\Gamma=\operatorname{Sub}(A) \cup\{\nabla \mathbf{\Delta} B \mid \mathbf{\Delta} B \in \operatorname{Sub}(A)\} \cup\{\mathbf{\Delta} \nabla B \mid \nabla B \in \operatorname{Sub}(A)\} .
$$

From this set, we can now define the set

$$
\begin{aligned}
\Sigma=\operatorname{Sub}(A) & \cup\left\{(\nabla \mathbf{\Delta})^{n} \nabla B \mid n \geq 0 \text { and } \nabla B \in \Gamma\right\} \\
& \cup\left\{\mathbf{\Lambda}(\nabla \mathbf{\Delta})^{n} \nabla B \mid n \geq 0 \text { and } \nabla B \in \Gamma\right\} \\
& \cup\left\{(\mathbf{\Delta} \nabla)^{n} \mathbf{\Delta} B \mid n \geq 0 \text { and } \mathbf{\Delta} B \in \Gamma\right\} \\
& \cup\left\{\nabla(\mathbf{\Delta} \nabla)^{n} \mathbf{\Delta} B \mid n \geq 0 \text { and } \mathbf{\Delta} B \in \Gamma\right\} .
\end{aligned}
$$

Obviously, $\operatorname{Sub}(A) \subseteq \Gamma \subseteq \Sigma$.

Lemma 3.1. (a) If $\nabla B \in \Sigma$, then $\Delta \nabla B \in \Sigma$.

(b) If $\mathbf{\Delta} B \in \Sigma$, then $\nabla \mathbf{\Delta} B \in \Sigma$.

Proof. (a) Suppose that $\nabla B \in \Sigma$. If $\nabla B$ is of the form $(\nabla \mathbf{\Lambda})^{n} \nabla C$ for some $n \geq 0$, where $\nabla C \in \Gamma$, then $\boldsymbol{\Delta} \nabla B=\mathbf{\Lambda}(\nabla \mathbf{\Delta})^{n} \nabla C$ belongs to $\Sigma$ by definition. If $\nabla B$ has the form of $\nabla(\boldsymbol{\Lambda} \nabla)^{m} \mathbf{\Lambda} C$ for some $m \geq 0$ where $\mathbf{\Delta} C \in \Gamma$, then by the definition, $\mathbf{\Delta} \nabla B=\boldsymbol{\Delta} \nabla(\mathbf{\Delta} \nabla)^{m} \mathbf{\Delta} C=(\mathbf{\Delta} \nabla)^{m+1} \mathbf{\Delta} C$ is in $\Sigma$.

Assertion (b) can be proved analogously.

A set of IntGC-formulas $\Sigma$ is said to be closed under subformulas if $B \in \Sigma$ and $C \in \operatorname{Sub}(B)$ imply $C \in \Sigma$.

Lemma 3.2. The set $\Sigma$ is closed under subformulas.

Proof. Let $B \in \Sigma$. If $B$ is of the form $C \vee D, C \wedge D, C \rightarrow D$, or $\neg C$, then $B$ must be in $\operatorname{Sub}(A)$ by the definition of $\Sigma$. Thus, $C, D \in \operatorname{Sub}(A) \subseteq \Sigma$.

If $\boldsymbol{\Delta} B \in \Sigma$ is of the form $\boldsymbol{\Delta}(\nabla \mathbf{\Delta})^{n} \nabla C$ for some $\nabla C \in \Gamma$ and $n \geq 0$, then $B=(\nabla \mathbf{\Lambda})^{n} \nabla C \in \Sigma$.

If $\boldsymbol{\Delta} B \in \Sigma$ has the form $(\boldsymbol{\Delta} \nabla)^{n} \boldsymbol{\Delta} C$ for some $n \geq 0$ and $\boldsymbol{\Delta} C \in \Gamma$, then $\mathbf{\Delta} B=\mathbf{\Delta}(\nabla \mathbf{\Delta})^{n} \nabla D$, since $\mathbf{\Delta} C \in \Gamma$ means that $C=\nabla D \in \operatorname{Sub}(A)$. Then, $B=(\nabla \mathbf{\Lambda})^{n} \nabla D \in \Sigma$.

The remaining two cases are proved analogously.

We now define for every formula $B \in \Sigma$, a unique formula $B^{*} \in \Gamma$ as follows:

(i) If $B \in \operatorname{Sub}(A)$ and $B$ is not of the form $\nabla C$ nor $\boldsymbol{\Delta} C$, then $B^{*}=B$.

(ii) If $B$ is of the form $(\nabla \mathbf{\Lambda})^{n} \nabla C$, where $\nabla C \in \Gamma$, then $B^{*}=\nabla C$.

(iii) If $B$ is of the form $\boldsymbol{\Lambda}(\nabla \mathbf{\Lambda})^{n} \nabla C$, where $\nabla C=\nabla \mathbf{\Delta} D \in \Gamma$ for some $\mathbf{\Delta} D \in \operatorname{Sub}(A)$, then $B^{*}=\mathbf{\Delta} D$.

(iv) If $B$ is of the form $(\boldsymbol{\Delta} \nabla)^{n} \mathbf{\Delta} C$, where $\boldsymbol{\Delta} C \in \Gamma$, then $B^{*}=\boldsymbol{\Delta} C$.

(v) If $B$ is of the form $\nabla(\boldsymbol{\Delta} \nabla)^{n} \mathbf{\Lambda} C$, where $\boldsymbol{\Delta} C=\boldsymbol{\Delta} \nabla D \in \Gamma$ for some $\nabla D \in \operatorname{Sub}(A)$, then $B^{*}=\nabla D$.

Related to the above definitions, we can write the following lemma.

Lemma 3.3. For every $B \in \Sigma$, there exists a unique $B^{*} \in \Gamma$ such that the formula $B \leftrightarrow B^{*}$ is provable in IntGC. 
Proof. We consider cases (ii) and (iii) only.

(ii) $B=(\nabla \mathbf{\Delta})^{n} \nabla C=(\nabla \mathbf{\Delta})^{n-1} \nabla \mathbf{\Delta} \nabla C \leftrightarrow(\nabla \mathbf{\Delta})^{n-1} \nabla C \leftrightarrow \cdots \leftrightarrow \nabla C=B^{*}$, because $\nabla C \leftrightarrow \nabla \boldsymbol{\Delta} \nabla C$ by (f2).

(iii) $B=\mathbf{\Delta}(\nabla \mathbf{\Lambda})^{n} \nabla C=\mathbf{\Delta} \nabla \mathbf{\Delta}(\nabla \mathbf{\Delta})^{n-1} \nabla C \leftrightarrow \mathbf{\Delta}(\nabla \mathbf{\Lambda})^{n-1} \nabla C \leftrightarrow \cdots \leftrightarrow$ $\mathbf{\Delta} \nabla C=\mathbf{\Delta} \nabla \mathbf{\Delta} D=\mathbf{\Delta} D=B^{*}$, since $\boldsymbol{\Delta} A \leftrightarrow \boldsymbol{\Delta} \nabla \mathbf{\Delta} A$ for any $A$.

Lemma 3.3 says that since $\Gamma$ is finite, also the set $\Sigma$ can be considered "finitary", because it can be divided into classes of provably equivalent formulas such that each class corresponds to one formula of $\Gamma$.

Now we define an equivalence $\sim$ on the set $X$ by setting

$$
x \sim y \Longleftrightarrow(\forall B \in \Sigma) x \models B \text { iff } y \models B .
$$

This means that points $x$ and $y$ are equivalent if they satisfy exactly the same formulas of $\Sigma$. We denote by $[x]$ the $\sim$-class of $x$, and $X / \sim$ is the set of all $\sim$-classes.

Lemma 3.4. The quotient set $X / \sim$ is finite.

Proof. Let $x \in X$. For all $y \in X,[x] \neq[y]$ means that there exists a formula $B \in \Sigma$ that "separates" $x$ and $y$, that is, either (i) $x \models B$ and $y \not \models B$, or (ii) $y \models B$ and $x \not \models B$. For instance, in case (i) this means by Lemma 3.3 that $x \models B^{*}, y \not \models B^{*}$, and $B^{*} \in \Gamma$. Because the set $\Gamma$ is finite, only a finite number of classes can be "separated" from $[x]$. Hence, also the quotient set $X / \sim$ must be finite.

We denote $X / \sim$ simply by $X^{f}$. We define in $X^{f}$ the relations $\leq^{f}$ and $R^{f}$ by setting:

$$
\begin{aligned}
{[x] \leq^{f}[y] } & \Longleftrightarrow(\forall B \in \Sigma) x \models B \text { implies } y \models B ; \\
{[x] R^{f}[y] } & \Longleftrightarrow(\forall B \in \Sigma) \nabla B \in \Sigma \text { and } y \models \nabla B \text { imply } x \models B .
\end{aligned}
$$

We can now write the following lemma.

Lemma 3.5. (a) If $x \leq y$, then $[x] \leq^{f}[y]$.

(b) If $x R y$, then $[x] R^{f}[y]$.

Proof. Claim (a) is obvious, because our Kripke frames are persistent.

(b) Assume $x R y, B \in \Sigma$, and $\nabla B \in \Sigma$. By Lemma 3.1, also $\boldsymbol{\Delta} \nabla B \in \Sigma$. If $y \models \nabla B$, then $x R y$ gives $x \models \boldsymbol{\Delta} \nabla B$. We have $x \models B$, because $\boldsymbol{\Delta} \nabla B \rightarrow B$ is a valid formula. Hence, $|x| R^{f}|y|$.

Lemma 3.6. The structure $\mathcal{F}^{f}=\left(X^{f}, \leq^{f}, R^{f}\right)$ is a Kripke frame.

Proof. It is clear that $\leq^{f}$ is a preorder. Therefore, it is enough to show that

$$
\left(\geq^{f} \circ R^{f} \circ \geq^{f}\right) \subseteq R^{f} .
$$

Suppose that $[x] \geq^{f}[y],[y] R^{f}[z]$, and $[z] \geq^{f}[w]$. For all $B \in \Sigma$, if $\nabla B \in \Sigma$ and $w \models \nabla B$, then $z \models \nabla B$ because $[z] \geq^{f}[w]$. Now $y \models B$ by $[y] R^{f}[z]$. Finally, $[x] \geq^{f}[y]$ implies $x \models B$. Thus, $[x] R^{f}[w]$,

Our next lemma gives another condition for $R^{f}$.

Lemma 3.7. $[x] R^{f}[y] \Longleftrightarrow(\forall B \in \Sigma) \Delta B \in \Sigma$ and $y \models B$ imply $x \models \Delta B$. 
Proof. Let $B \in \Sigma$. Assume $[x] R^{f}[y], \mathbf{\Delta} B \in \Sigma$ and $y \models B$. Since $B \rightarrow \nabla \mathbf{\Delta} B$ is a provable formula, we have $y \models B \rightarrow \nabla \mathbf{\Delta} B$ and so $y \models \nabla \mathbf{\Delta} B$. Because $\mathbf{\Delta} B \in \Sigma$, Lemma 3.1 gives $\nabla \mathbf{\Delta} \in \Sigma$. Since $[x] R^{f}[y]$, we get $x \models \mathbf{\Delta} B$.

Conversely, assume that the right-side of the condition holds. If $\nabla B \in \Sigma$ and $y \models \nabla B$, then by Lemma 3.1, $\mathbf{\Delta} \nabla B \in \Sigma$, from which we get $x \models \boldsymbol{\Delta} \nabla B$ by the assumption. Because $\boldsymbol{\Delta} \nabla B \rightarrow B$ is a provable formula, we have $x \models B$. Thus, $[x] R^{f}[y]$.

We define the valuation $v^{f}$ in such a way that for all proposition variables $p \in \Sigma:$

$$
v^{f}(p)=\{[x] \mid x \models p\} .
$$

Then, $\mathcal{M}^{f}=\left(X^{f}, \leq^{f}, R^{f}, v^{f}\right)$ is called filtration of $\mathcal{M}$ through $\Sigma$.

Lemma 3.8. For any $B \in \Sigma$ and $x \in X, x \models B$ iff $[x] \models B$.

Proof. By induction on $B$. This can be done, because $\Sigma$ is closed under subformulas. The base case follows immediately from the definition of $v^{f}$, and with respect to $\vee$ and $\wedge$ the proof is obvious.

(i) Let $B$ of the form $\neg C \in \Gamma$. Assume $[x] \models \neg C$. If $x \not \models \neg C$, then there exists $y \geq x$ such that $y \models C$. Since $\Gamma$ is closed under subformulas, also $C \in \Gamma$ and $[y] \models C$ by the induction hypothesis. Because $y \geq x$, we have $[y] \geq^{f}[x]$ by Lemma 3.5. This gives that $[x] \not \models \neg C$, a contradiction. So, $x \models \neg C$.

Conversely, suppose that $x \models \neg C$. Because $C \in \Gamma$, then by the definition, $[y] \geq^{f}[x]$ implies $y \models \neg C$ and $y \not \models C$. By the induction hypothesis, we have that $[y] \geq^{f}[x]$ implies $[y] \not \models C$, that is, $[x] \models \neg C$.

(ii) Let $B$ of the form $C \rightarrow D \in \Gamma$. Assume $x \models C \rightarrow D$ and $[x] \not \models$ $C \rightarrow D$. Then, there exists $[y] \geq^{f}[x]$ such that $[y] \models C$, but $[y] \not \models D$. By induction hypothesis, $y \models C$ and $y \not \models D$. Therefore, $y \not \models C \rightarrow D$, which is impossible because $[y] \geq^{f}[x]$. Thus, $[x] \models C \rightarrow D$.

On the other hand, if $[x] \models C \rightarrow D$, then for all $[y] \geq^{f}[x],[y] \models C$ implies $[y] \models D$. If $x \not \models C \rightarrow D$, there exists $y \geq x$ such that $y \models C$ and $y \not \models D$. Now $y \geq x$ gives $[y] \geq^{f}[x]$, and $[y] \models C$ and $[y] \not \models D$ by the induction hypothesis. But this is impossible. So, $x \models C \rightarrow D$.

(iii) Let $B$ be of the form $\nabla C \in \Gamma$. Assume that $x \models \nabla C$. If $[y] R^{f}[x]$, then $y \models C$, and $[y] \models C$ follows from the induction hypothesis. Hence, $[x] \models \nabla C$.

Conversely, assume that $[x] \models \nabla C$ and $y R x$. Then, $[y] R^{f}[x]$ by Lemma 3.5, which gives $[y] \models C$. We obtain $y \models C$ by the induction hypothesis, and so $x \models \nabla C$.

(iv) Let $B$ be of the form $\boldsymbol{\Delta} C \in \Gamma$. If $x \models \boldsymbol{\Delta} C$, then there exists $y$ such that $x R y$ and $y \models C$. By the induction hypothesis, $[y] \models C$. Since $x R y$, we have $[x] R^{f}[y]$ and $[x] \models \boldsymbol{\Lambda} C$.

On the other hand, if $[x] \models \boldsymbol{\Lambda} C$, then there exists $y$ such that $[x] R^{f}[y]$ and $[y] \models C$. This implies $y \models C$ by the induction hypothesis. By Lemma 3.7, we get $x \models \boldsymbol{\Lambda} C$.

Finally, we may write the following proposition.

Proposition 3.9. IntGC has the finite model property and is decidable. 
Proof. Suppose that a formula $A$ is not provable. Then, there exists a model $\mathcal{M}=(X, \leq, R)$ such that $A$ is not valid in $\mathcal{M}$. This means that there exists $x \in X$ such that $x \not \forall A$. We may define the set $\Sigma$ and the filtration of $\mathcal{M}$ through $\Sigma$ as above. Because $A \in \Sigma$, then $[x] \not \models A$ by Lemma 3.8, and hence $A$ is not valid in the finite model $\mathcal{M}^{f}$.

In addition, it is well known that if a logic is finitely axiomatised with the finite model property, then the logic is decidable.

\section{REFERENCES}

[1] Wojciech Dzik, Jouni Järvinen, and Michiro Kondo, Intuitionistic propositional logic with Galois connections, Logic Journal of the IGPL 18 (2010), 837-858.

\section{Contact Addresses}

WD: Institute of Mathematics, University of Silesia, ul. Bankowa 12 , 40-007 Katowice, Poland

E-mail address: dzikw@silesia.top.pl

JJ: HTTP://SITES.GOOGLE.COM/SITE/JOUNIKALERVOJARVINEN/

E-mail address: Jouni.Kalervo.Jarvinen@gmail.com

MK: School of Information Environment, Tokyo Denki University, Inzai, 270-1382, JAPAN

E-mail address: kondo@sie.dendai.ac.jp 